\title{
MAXWELL: EL CULTIVAR DE TRIGO QUE HA SUPERADO LAS BARRERAS PRODUCTIVAS EN EL SUR DE CHILE
}

\section{MAXWELL: THE WHEAT CULTIVAR THAT HAS OVERCOME THE YIELD BARRIERS IN SOUTHERN CHILE}

\author{
Claudio Jobet ${ }^{1,2^{*}}$, Ricardo Madariaga ${ }^{1}$, Iván Matus ${ }^{1}$, Ricardo Campillo ${ }^{1}$, y Javier Zuñiga ${ }^{1}$ \\ ${ }^{1}$ Instituto de Investigaciones Agropecuarias (INIA), Centro Regional Carillanca, Casilla 58-D, Temuco, \\ Chile. \\ 2 Universidad de La Frontera, Facultad de Agronomía, Casilla 54-D, Temuco, Chile. \\ * Autor para correspondencia E-mail: cjobet@inia.cl
}

\section{RESUMEN}

Maxwell es un cultivar de trigo (Triticum aestivum L.) de invierno, introducido a Chile en el año 2004 por el Instituto de Investigaciones Agropecuarias (INIA) en colaboración con la Empresa Saaten Union Research de Francia, y liberado comercialmente el año 2012. Maxwell fue evaluado por el Programa de Mejoramiento de Trigo del INIA de Chile. Esta investigación se realizó con el fin de evaluar su comportamiento agronómico en el área nacional más importante de producción de trigo, bajo diferentes condiciones de suelo y clima. En base a los resultados obtenidos se pudo establecer que para el hábito de crecimiento, altura de la planta, precocidad, sanidad, rendimiento y calidad industrial, el cultivar Maxwell se destacó por sobre los testigos comerciales y mostró gran adaptabilidad para siembras tempranas, en zonas de inviernos largos y primaveras húmedas. La planta alcanza una altura promedio de $90 \mathrm{~cm}$, siendo clasificado como un trigo enano a semienano. Su rendimiento promedio, en diferentes localidades y varios años de evaluación, fue $1,06 \mathrm{t} \mathrm{ha}^{-1}$, superior al testigo, siendo clasificado desde el punto de vista industrial como un trigo de calidad intermedia.

Palabras clave: cultivar, trigo invierno, rendimiento, sintético

\section{ABSTRACT}

Maxwell is a winter wheat (Triticum aestivum L.) cultivar. It was introduced in Chile in 2004 by the National Institute of Agricultural Research (INIA) with the collaboration of the Saaten Union Research Company of France, and released in 2012. Maxwell was evaluated under the Wheat Plant Breeding Program of the INIA (Chile). This study was carried out in the main wheat production area of Chile under different soil types and climatic conditions. The results indicate that Maxwell was superior to commercial checks in terms of growth habit, plant height, precocity, disease resistance, yield and bread-making quality. It also showed better adaptability to early sowing in agro-climatic zones with long winters and humid springs. The adult plant reached a mean height of $90 \mathrm{~cm}$, so that Maxwell can be classified as a semi-dwarf or dwarf wheat. The mean grain yield was higher than the control, reaching a value of $1.06 \mathrm{t} \mathrm{ha}^{-1}$ for the different locations and years of evaluation, and from an industrial point of view can be considered as a medium-quality wheat .

Key words: cultivar, winter wheat, yield, synthetic.

Recibido: 27 mayo 2016. Aceptado: 12 agosto 2016. 


\section{ORIGEN, CRUZA Y PEDIGRI}

Maxwell es una variedad de trigo (Triticum aestivum L.) que se puede utilizar en la industria molinera, galletera y panadera, perteneciente a la Empresa Saaten Union Research de Francia. Fue introducido a Chile en un jardín $\mathrm{F}_{9 ;}$ se recibió el año 2004 con la denominación interna EssaiSUR.2001/008. Maxwell es producto de una cruza compuesta realizada en el programa de trigos híbridos de esta empresa, siendo sus progenitores cinco genotipos elite mas dos líneas sintéticas mejoradas derivadas del Triticum dicoccum (AABB), especie ancestral del Triticum durum (AABB) y de Triticum aestivum (AABBDD), las cuales se utilizaron como fuente parental. El material, sintético mejorado, provino de la Universidad de Wageningen en Holanda, el cual se originó a partir de cruzas amplias con T. dicoccum (Emmer) con el fin de incluir, preferentemente, genes de resistencia a enfermedades en trigo (Fig. 1).

Estas líneas sintéticas portan en su pedigrí genes específicos para roya estriada [agente causal Puccinia striiformis] ( $Y r$ 1, 5 y 6), genes de resistencia para roya de la hoja [agente causal Puccinia triticina] (Lr 9 y 24), mas algunos genes de resistencia a oidio [agente causal Blumeria graminis] ( $M d c, 12$ y 13) (Saaten Union Research. Comunicación personal). La caracterización de virulencias, realizada en la Universidad de Washington en las que participaron muestras de roya estriada de Chile, confirmaron la alta efectividad del gene Yr 5, el cual se encontraría presente en Maxwell, y de otros genes como YrSp y Yr15 (Sharma-Poudyal et al., 2013). Mediante el monitoreo anual que se realiza en INIA CRI Quilamapu con las isolineas para Puccinia triticina se puede concluir en la temporada agrícola 2015-16 que Lr9 presente en NIL-Thatcher-Lr9Transfer y Lr24 en NIL-Thathcher-Lr 24-Agent no confieren protección completa a la roya de la hoja. Sin embargo la acción conjunta de estos genes presentes en Maxwell, así como de otros posiblemente existentes y no descritos, le permiten expresar solo moderada susceptibilidad a roya de la hoja. Adicionalmente se incluye en estas líneas la banda de gluteninas de alto peso molecular $6,1+22$ presentes en el cromosoma $1 \mathrm{~B}$, lo que les da un mayor valor en el tenor de proteína del grano y por ende mejor calidad industrial panadera.

Estas líneas conformaron el compuesto (machos) que se cruzaron con líneas elite (hembras) del programa de trigo de la Saaten Union Research en Francia, las cuales se "castraron" vía gametocida (Croisor) permitiendo la libre fecundación de tipo cruzada. Las progenies resultantes fueron varias veces retrocruzas con material elite, seleccionando

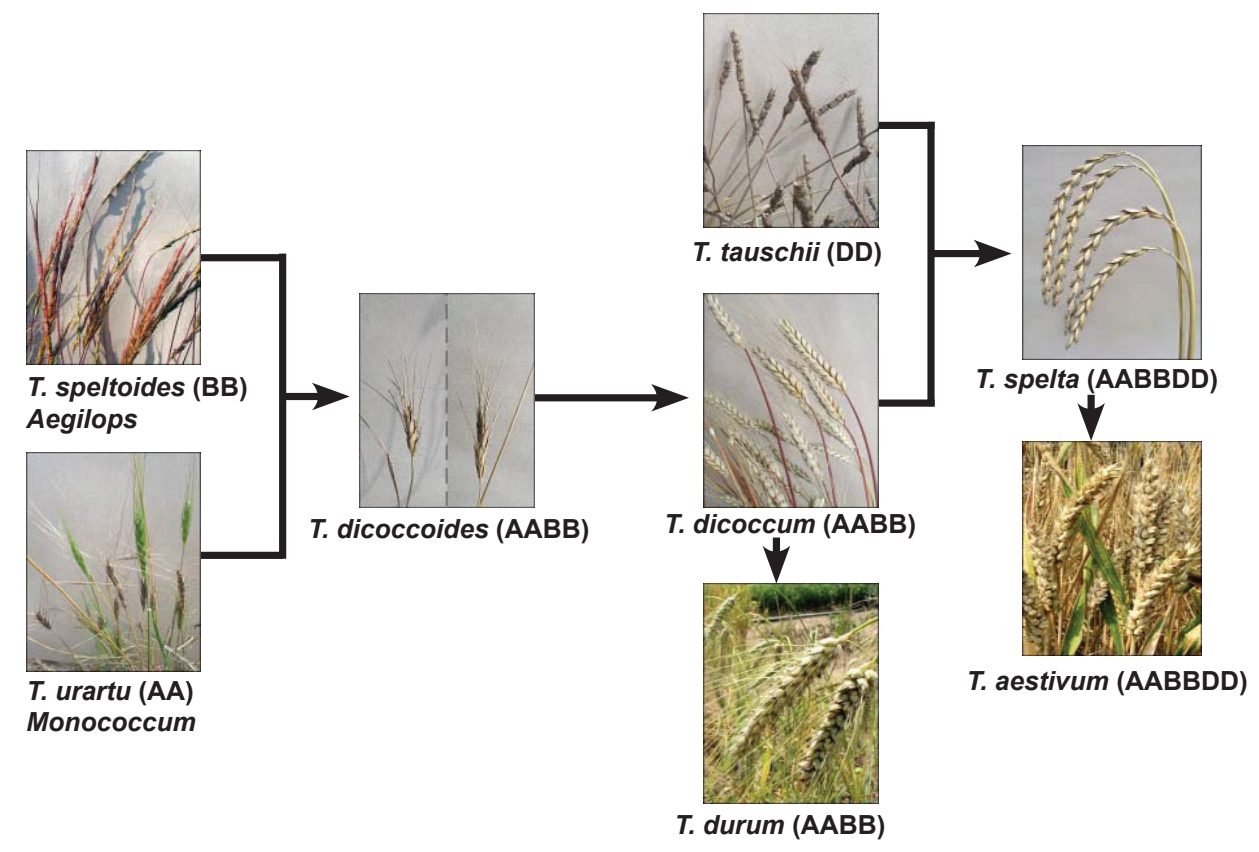

Fig. 1. Desarrollo y evolución del trigo cultivado.

Fuente: Dubcovsky y Dvorak, 2007.

Fig. 1. Development and evolution of cultivated wheat.

Source: Dubcovsky y Dvorak, 2007. 
por tipo agronómico, resistencia y rendimiento. Estas líneas finalmente fueron sometidas a la homogenización cromosomal por medio de la cruza inter especifica trigo x maíz, obteniendo dobles haploides (Jobet et al., 2003), de entre los cuales se seleccionó finalmente la variedad Maxwell, inscribiéndose, en el Sistema de Registro de Plantas de Europa en Inglaterra (2006) y en el 2007 en Francia (Mayores antecedentes en www. cpvo.fr)

En Chile, esta variedad ha sido evaluada desde el año 2005 en ensayo de rendimiento en Vilcún, INIA Carillanca $\left(38^{\circ} 50^{\prime} \mathrm{S}, 72^{\circ} 25^{\prime} \mathrm{O}\right)$ destacándose por su tipo agronómico, por su adaptabilidad a zonas de siembras tempranas e inviernos largos, por su alto nivel productivo y por su buen comportamiento fitosanitario, incorporándose a los ensayos estándares nacionales a partir del año 2006, en las localidades de Chillán (36 $31^{\prime}$ S, $\left.71^{\circ} 54^{\prime} \mathrm{O}\right)$, Santa Bárbara $\left(37^{\circ} 40^{\prime} \mathrm{S}, 72^{\circ} 01^{\prime} \mathrm{O}\right)$, Quino ( $\left.38^{\circ} 49^{\prime} \mathrm{S}, 72^{\circ} 38^{\prime} \mathrm{O}\right)$, Máfil ( $35^{\circ} 39^{\prime} \mathrm{S}, 7^{\circ} 02^{\prime}$ O) y Purranque $\left(40^{\circ} 51^{\prime} S, 73^{\circ} 12^{\prime} \mathrm{O}\right)$, lo que da un total de seis sitios, los cuales representaron a cuatro regiones del centro sur y sur de Chile, donde se concentra mas del $80 \%$ de la superficie sembrada de trigo en el país (ODEPA, 2016).

\section{DESCRIPCIÓN MORFOLÓGICA DE LA PLANTA}

Es un trigo de hábito de desarrollo invernal (requiere vernalización), muy rastrero al estado de plántula, de macollaje débil en sus inicios y de lento crecimiento. Sus hojas son de color verde oscuro, con hoja bandera delgada y semierecta. La espiga es de color blanco amarillo, semi curvada a la madurez, forma paralela, muy densa, compacta y con barbas. El grano es de forma redondeada, tamaño mediano, color café oscuro, pudiendo presentar ocasionalmente ciertos cambios de pigmentación en el endosperma, dependiendo del ambiente y/o temporada. La altura de planta adulta varía entre 70 y $95 \mathrm{~cm}$, con un promedio de $90 \mathrm{~cm}$., siendo considerado un trigo semienano a enano, y significativamente más bajo que los cultivares Dollinco-INIA, Kumpa-INIA y TukanINIA. Tiene un tallo hueco de longitud media con una muy buena resistencia a la tendedura, superior a la mayoría de las variedades de trigo invernales actualmente recomendadas

\section{CARACTERISTICAS FITOPATOLÓGICAS}

Este cultivar se destaca por su resistencia a polvillo estriado (Puccinia striiformis West. f. sp. tritici Erikss.), sin embargo, a través de los años, ha ido presentado moderada susceptibilidad a polvillocoloradodelahoja. Estudios comparativos de respuesta a la aplicación de fungicidas al follaje, ciclo agrícola 2015-16, indicaron que el cv. Maxwell respondió solamente con $0,43 \mathrm{t} \mathrm{ha}^{-1}$ no siendo estadísticamente significativo con $\mathrm{Pr}>\mathrm{F}$ de 0,5892 y CV de 14,3\%. (Madariaga et al., 2016. Datos no publicados). Tiene buena tolerancia a septoriosis, es resistente a oidio y muestra susceptibilidad a enfermedades radiculares como mal de pie (Gaeumannomycea graminis var. tritici), por lo que se recomienda sembrarla en rotaciones adecuadas y en suelos libres de esta enfermedad. Sus reacciones de resistencia y susceptibilidad (Saari, y Prescott, 1975; Peterson et al., 1984; Stubbs et al., 1986), a diversas enfermedades que atacan al trigo esta muy relacionada a su estructura genética, y según Mujeeb-Kazi y Hettel (1995), parte importante en su expresión esta dada por genes provenientes de uno de sus progenitores, el Triticum dicoccum (AABB).

\section{RENDIMIENTO DE GRANO}

El promedio de rendimiento para los años evaluados (2005 al 2015) y para las diferentes

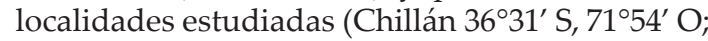
Santa Bárbara $37^{\circ} 40^{\prime} \mathrm{S}, 72^{\circ} 01^{\prime} \mathrm{O}$; Quino $38^{\circ} 49^{\prime} \mathrm{S}$,

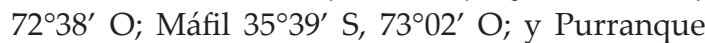
$40^{\circ} 51^{\prime}$ S, $73^{\circ} 12^{\prime}$ O), fue significativamente superior $(\mathrm{P}<0,05)$ a Kumpa-INIA, cultivar de invierno que ha sido uno de los más sembrados por su adaptabilidad a esas condiciones y a su gran potencial de rendimiento. La diferencia más notable entre Maxwell y este cultivar de invierno utilizado como testigo, se presenta en la localidad de Quino (1,53 t ha $\left.{ }^{-1}\right)$, sin embargo en todas las otras localidades, Maxwell superó al testigo (Tabla 1), alcanzando un promedio, de localidades y años de evaluación, de 11,98 $\mathrm{t} \mathrm{ha}^{-1}$ contra 10,92 $\mathrm{t}$ ha $^{-1}$ de Kumpa INIA. Esta situación refleja el gran potencial productivo de este cultivar, lo que sin duda hace un importante aporte a la producción de trigo nacional. En base a los resultados observados, Maxwell ha demostrado tener un rendimiento estable y superior a muchas variedades comerciales y líneas avanzadas, dentro de una gran zona triguera con diversas características agroclimáticas.

\section{CALIDAD INDUSTRIAL}

El cultivar Maxwell es un trigo de textura de grano dura a semidura, presenta un porcentaje de proteína promedio superior al $10,0 \%$, una cifra de sedimentación de alrededor de $38 \mathrm{~cm}^{3}$, y su contenido de gluten húmedo puede ser cercano a un $27 \%$, variando de 23 a $31 \%$ o mas, dependiendo de la localidad y del manejo de la fertilización, que lo ubican, según el Instituto 
Tabla 1. Rendimiento promedio de grano $\left(\mathrm{t} \mathrm{ha}^{-1}\right)$ del cv. Maxwell comparada con el cv. Kumpa-INIA en seis localidades durante varias temporadas (2005/12 al 2014/15).

Table 1. Mean grain yield ( $\mathrm{tha} \mathrm{h}^{-1}$ ) of $\mathrm{cv}$. Maxwell compared to $\mathrm{cv}$. Kumpa-INIA in six experimental sites over several seasons $(2005 / 12$ to $2014 / 15)$.

\begin{tabular}{|c|c|c|c|c|c|c|}
\hline \multirow[t]{2}{*}{ Localidad } & \multirow[t]{2}{*}{ Variedad } & \multicolumn{4}{|c|}{ Rendimiento } & \multirow[b]{2}{*}{ Promedio } \\
\hline & & $2005 / 12$ & $2012 / 13$ & $2013 / 14$ & $2014 / 15$ & \\
\hline \multirow{3}{*}{ Chillán } & & --------- & th & 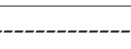 & ---------- & \\
\hline & Maxwell & 10,80 & $10,96^{*}$ & $14,41^{*}$ & $11,20^{*}$ & 11,84 \\
\hline & Kumpa-INIA & 10,34 & 9,47 & 12,10 & 9,73 & 10,41 \\
\hline \multirow[t]{2}{*}{ Sta. Bárbara } & Maxwell & 5,69 & $11,96^{*}$ & 5,41 & 8,72 & 7,95 \\
\hline & Kumpa-INIA & 3,94 & 9,95 & 4,12 & 8,13 & 6,53 \\
\hline \multirow[t]{2}{*}{ Quino } & Maxwell & 10,01 & $18,31^{*}$ & $12,12^{*}$ & $16,59^{*}$ & 14,26 \\
\hline & Kumpa-INIA & 9,71 & 16,71 & 11,02 & 13,48 & 12,73 \\
\hline \multirow[t]{2}{*}{ Vilcún } & Maxwell & 10,36 & 7,72 & 13,26 & 12,21 & 10,89 \\
\hline & Kumpa-INIA & 9,54 & 9,02 & 14,21 & 11,38 & 11,38 \\
\hline \multirow[t]{2}{*}{ Máfil } & Maxwell & 10,41 & $14,49^{*}$ & $13,21^{*}$ & $13,56^{*}$ & 12,92 \\
\hline & Kumpa-INIA & 11,58 & 12,35 & 11,80 & 11,85 & 11,90 \\
\hline \multirow[t]{2}{*}{ Purranque } & Maxwell & 15,07 & $12,20^{*}$ & $17,00^{*}$ & 11,81 & 14,02 \\
\hline & Kumpa-INIA & 11,86 & 8,92 & 15,42 & $13,89^{*}$ & 12,52 \\
\hline
\end{tabular}

* Los valores promedios de los tratamientos, por variedad y localidad, indican diferencias estadísticamente significativas según la prueba de $\mathrm{T}(\mathrm{P}<0,05)$.

Nacional de Normalización (INN, 2000), en la categoría de trigo intermedio (NCh 1237-2000). Sus características alveográficas son aceptables, con un valor $\mathrm{W}$ promedio de 183, con cierto grado de tenacidad de la masa $(\mathrm{P} / \mathrm{L}>1)$, volumen de pan cercano a los $500 \mathrm{~cm}^{3}$ y miga de color crema. Es un trigo que puede ser utilizado para mezcla para la elaboración de harina de pan, en la industria de la galletería y repostería. De acuerdo a los resultados de calidad, este cultivar se comporta mas como un trigo panadero de tipo intermedio, pudiendo clasificar incluso como trigo fuerte cuando el porcentaje de gluten húmedo supera el $30 \%$ (Tabla 2).

Los resultados de calidad (Tabla 2), incluyen evaluaciones de ensayos realizados desde Chillán hasta Purranque, para la temporada 2013 y 2014. Se destacan valores para gluten húmedo y sedimentación, en algunas localidades, como Quino, Máfil y Purranque, logrando niveles que pueden ser considerados efectivamente como trigo fuerte (Datos no presentados). Adicionalmente, en la Unidad de Biotecnología del INIA Carillanca, se ha medido el color en términos de parámetros de espacio de color CIELAB de índice de color (Oliver et al., 1993), el cual arrojó los siguientes resultados: $\mathrm{L}^{*}=$ 91,$7 ; \mathrm{b}^{*}=10,9$, e IC $=80,6$ (nivel promedio), lo que implica que Maxwell produce harina color crema, la cual se puede "blanquear" con peroxido benzoilo a nivel de molino (Zúñiga, J.
2015. INIA Carillanca. Comunicación personal). Por el contrario, la variedad Kumpa INIA produce una harina mas oscura (color ceniza, $b^{*}$ $=11,5$ ), posiblemente por el porte de segmento heterólogo derivado del centeno (translocación), lo que ha provocado un cierto rechazo de la variedad por parte de la industria, y cuyo color de harina es difícil de blanquear.

\section{PERFIL ELECTROFORETICO DE GLUTENINAS DE ALTO PESO MOLECULAR}

De acuerdo al análisis electroforético establecida por Payne (1987) y Shewry et al. (1992), Maxwell contiene el alelo nulo en el locus Glu-1A ubicado en el brazo largo del cromosoma 1A, los alelos $7+8$ en el locus Glu1B, ubicado en el brazo largo del cromosoma $1 \mathrm{~B}$ y los alelos $5+10$ en el genoma D. El score para estas bandas es de 8 , lo cual lo clasifica como una variedad intermedia en cuanto a la calidad de sus proteínas, siendo el mínimo 4 y el máximo 10.

\section{ANALISIS MOLECULAR}

Maxwell contiene el alelo de dureza PIN $\mathrm{a}(\mathrm{b})$, identificados por medio de PCR (Reacción en cadena de polimerasa), lo que se confirma con el índice de dureza obtenido (Tabla 2), y no presenta elemento heterólogo de centeno 
Tabla 2. Características de calidad del cv. Maxwell comparada con el testigo cv. Kumpa-INIA. Rangos promedios observados para diferentes localidades en dos temporadas.

Table 2. Quality characteristics of cv. Maxwell compared to control cv. Kumpa-INIA. Mean values measured in different locations and two seasons.

\begin{tabular}{|c|c|c|c|c|c|c|}
\hline & \multicolumn{3}{|c|}{ Maxwell } & \multicolumn{3}{|c|}{ Kumpa INIA } \\
\hline & 2012/13 & 2013/14 & Prom. & $2012 / 13$ & 2013/14 & Prom. \\
\hline Indice de dureza, $\%{ }^{(1)}$ & 17,5 & 18,6 & 18,1 & 20 & 18,7 & 19,4 \\
\hline Peso del hectólitro, $\mathrm{kg} \mathrm{hL}^{-1}$ & 79,0 & 79,0 & 79,0 & 81 & 81 & 81,0 \\
\hline Sedimentación Zeleny, $\mathrm{cm}^{3(2)}$ & 37,3 & 39,1 & 38,2 & 38 & 42,4 & 40,2 \\
\hline Proteína (N x 5,7), \% base seca & 11,1 & 10,1 & 10,6 & 11,4 & 11,0 & 11,2 \\
\hline Valor $W^{(3)}$ & 187 & 179 & 183 & 168 & 212 & 190 \\
\hline $\mathrm{P}(\mathrm{m} / \mathrm{m})$ & 100 & 78 & 89 & 84 & 76 & 80 \\
\hline $\mathrm{L}(\mathrm{m} / \mathrm{m})$ & 71 & 88 & 80 & 83 & 83 & 83 \\
\hline $\mathrm{P} / \mathrm{L}$ & 1,5 & 0,96 & 1,2 & 1,0 & 1,0 & 1,0 \\
\hline Volumen de pan, $\mathrm{cm}^{3(4)}$ & 500 & 510 & 505 & 525 & 520 & 523 \\
\hline Color miga & Crema & Crema & Crema & Ceniza & Ceniza & Ceniza \\
\hline Gluten húmedo, \% ${ }^{(5)}$ & 28,0 & 31,4 & 26,7 & 29,0 & 28,6 & 28,8 \\
\hline Gluten index & 76 & 61 & 68,5 & 77 & 76 & 76,5 \\
\hline Falling Number, seg ${ }^{(6)}$ & 411 & 386 & 399 & 436 & 409 & 423 \\
\hline
\end{tabular}

Laboratorio de Calidad de Trigo, INIA Carillanca, Granotec Chile, Molino San Cristóbal, Santiago.

(1) Índice de dureza: Valores de 16 a 25 corresponden a un grano duro; valores de 26 a 30 corresponden a un grano semiduro. Valores de 31 o más corresponden a un grano blando.

(2) Sedimentación Zeleny: Cifras de 17 a $26,9 \mathrm{~cm}^{3}$ corresponden a un trigo suave; cifras de 27 a $32,9 \mathrm{~cm}^{3}$ corresponden a un trigo intermedio; cifras de 33 a 44 cc corresponde a un trigo fuerte; cifras superiores a 44 cc corresponden a un trigo muy fuerte.

(3) Valor W (alveograma): Menor de 150 corresponde a un valor bajo; entre 150 a 200 corresponde a un valor medio; entre 200 a más corresponde a un valor bueno.

(4) Volumen de pan: Menor de 550 corresponde a un valor bajo; entre 550 y 600 corresponde a un valor bueno; valores mayores a 600 corresponden a un valor muy bueno.

(5) Gluten húmedo: Igual o mayor a 30\% corresponde a trigo fuerte; entre 29,9 y 25,0\% corresponde a un trigo intermedio; contenido mínimo de $18,0 \%$ corresponde a un trigo suave.

(6) Falling Number: Índice de caída: Sobre 300 baja presencia de enzima alfa amilasa, no hay brotación; bajo 170 indica alta presencia de enzima alfa amilasa, por lo tanto actividad de brotación.

(translocación), identificado también mediante PCR (D'Oviedo y Anderson, 1995). Este último elemento, la presencia de la translocación 1BL.1RS, que es la mas común, se ha asociado con defectos en la calidad industrial del trigo, incluyendo la producción de masas pegajosas, propiedades de mezcla inferiores, bajo volumen de sedimentación y fermentación deficiente en trigo harinero ( $\mathrm{Li}$ et al., 2016) y bajo volumen de microsedimentación en trigo durum (Oak y Tamhankar, 2016)., Por otro lado, el segmento heterólogo derivado del centeno también puede producir un efecto de oscurecimiento de harina en diferentes niveles afectando de manera diferentes la expresión de la calidad industrial (Graybosch, 2001; Zhao et al., 2012)

\section{ZONA DE CULTIVO Y FECHAS DE SIEMBRA}

Maxwell se recomienda desde la región del Biobío hasta la Región de los Lagos, especialmente para suelos trumaos y transicionales, en localidades de inviernos largos y primaveras húmedas. Por su ciclo se recomienda sembrarlo desde abril hasta julio, aun cuando retrasando la época de siembra su potencial productivo tiene a disminuir. Por sus características agronómicas y buen potencial de rendimiento demostrado podría ocupar un lugar preferencial dentro de los trigos utilizados por los agricultores en el sur y centro sur de Chile. 


\section{CONCLUSIONES}

El cultivar Maxwell, es producto de una cruza compuesta, donde sus progenitores portan genes de especies ancestrales como Triticum dicoccum (AABB), lo que permite la presencia de una mayor diversidad genética en su patrimonio genético. Es un trigo que se ha adaptado a la zona triguera del sur y centro sur del país, con excelente nivel productivo y calidad industrial.

\section{LITERATURA CITADA}

Dubcovsky, J., and J. Dvorak. 2007. Genome plasticity: a key factor in the success of polyploid wheat under domestication. Science 316(5833):1862-1866.

D'Oviedo, R., and O. Anderson. 1995. PCR analysis to distinguish between alleles of a member of a multigene family correlated with bread-making quality. Theor. Appl. Genet. 88:759-763.

Graybosch, R.A. 2001. Uneasy unions: quality effects of rye chromatin transfers to wheat. Journal of Cereal Science 33(1):3-16

INN. 2000. Trigo harinero: Requisitos. Norma Chilena Oficial $\mathrm{N}^{\circ} 1237$ Of. 2000 (NCh 1237: of 2000). 16 p. Instituto Nacional de Normalización (INN), Santiago, Chile.

Jobet, C., J. Zúñiga, y H. Campos de Quiroz. 2003. Plantas dobles haploides generadas por cruza intergenérica de trigo x maíz. Agricultura Técnica 63(3):323-328.

Li, Z., T. Ren, B. Yan, F. Tan, M. Yang, and Z. Ren 2016. A mutant with expression deletion of gene Sec-1 in a 1RS.1BL line and its effect on production quality of wheat. PLoS ONE 11(1):e0146943. doi:10.1371/journal. pone. 0146943

Mujeeb-Kazi, A., and G. Hettel EDS. 1995. Utilizing wild grass biodiversity in wheat improvement: 15 year of wide cross research at CIMMYT. 140 p. Research Report № 2. CIMMYT, México, D.F., México.
ODEPA. 2016. Cultivos anuales: superficie, producción y rendimientos. Oficina de Estudios y Políticas Agrarias (ODEPA), Santiago, Chile.

Oak, M.D., and S.A. Tamhankar. 2016. 1BL/1RS translocation in durum wheat and its effect on end use quality traits. J. Plant Biochem. Biotechnol. DOI: 10.1007/s13562-016-0366-6

Oliver, J., A. Blakeney, and H. Allen. 1993. The colour of flour streams related to ash and pigment contents. Journal of Cereal Science 17(2):169-182.

Payne, P. 1987. Genetics of wheat storage proteins and the effect of allelic variation on breadmaking quality. Ann. Rev. Plant. Physiol. 38:141-153.

Peterson, R., A.B. Campbell, and A. E. Hannah. 1984. A diagrammatic scale for estimating rust intensity of leaves and stem of cereals. Can. J. Res. Sect. 26:496-500.

Saari, E., and J.M. Prescott. 1975. A scale for appraising the foliar intensity of wheat diseases. Plant Dis. Reporter 59:377-380.

Sharma-Poudyal, D., X.M. Chen, A.M. Wan, G.M. Zhan, Z.S. Kang, S.Q. Cao, et al. 2013. Virulence characterization of international collections of the wheat stripe rust pathogen, Puccinia striiformis f. $\mathrm{sp}$ tritici. Plant Disease 97(3):379-386.

Shewry, P., N. Halford, and A. Tathman. 1992. Critical review article. High molecular weight subunits of wheat glutenin. J. Cereal Sci. 15:105-120.

Stubbs, R.W., J.M. Prescott, E.E. Saari, and H.J. Dubin. 1986. Cereal disease methodology manual. Centro Internacional de Mejoramiento de Maiz y Trigo (CIMMYT), México.

Zhao C., F. Cui, X. Wang, S. Shan, X. Li, Y Bao, et al. 2012. Effects of 1BL/1RS translocation in wheat on agronomic performance and quality characteristics. Field Crops Research 127(27):79-84. 